\title{
Movie Microblog Marketing Based on the UID Deployment Model
}

\author{
Jin Zeng ${ }^{1,}$, Xiaonan Jin $^{1, b}$, Baotian Dong ${ }^{1, c}$ and Chunjiao Dong ${ }^{2, d}$ \\ ${ }^{1}$ School of Traffic and Transportation, Beijing Jiaotong University, 100044, China; \\ ${ }^{2}$ Center for Transportation Research, the University of Tennessee,37996, USA. \\ ajzeng@bjtu.edu.cn, b13120972@bjtu.edu.cn, 'btdong@bjtu.edu.cn, dcdong5@utk.edu
}

Keywords: movie, microblog marketing, UID deployment model

\begin{abstract}
With the rapid development of new media, a social media microblog platform has played an important role in the movie marketing. Compared with the ordinary screening model of the current movie microblog marketing, this paper established a UID(User Information Define) deployment model to achieve high exposure and high conversion by the choice of the crowd and appropriate materials delivery. The precision marketing examples of "Breakup Buddies" in Sina Weibo Data Center validates the rationality and availability of the model. The model provides theoretical basis and recommendations for microblog enterprise to develop new delivery system and improve existing delivery processes.
\end{abstract}

\section{Introduction}

As of June 2015, China's microblog users scale of 204 million and Internet utilization rate is $30.6 \%$. Microblogging has become one of the most enthusiastic ways of network transmission [1]. In this context, microblog showed huge commercial value by a great number of the movie business and led to a lot of successful movie marketing through the microblog platform. Microblog marketing was a force to be reckoned with. However, how to aim at ads and material on the basis of the target users and clever marketing strategy needed careful consideration.

Microblog marketing research overseas mainly focused on the influence factors, mode of transmission and the marketing effect. Yaroslav Ryabov [2] had carried on an empirical research of the influence factors of the microblogging marketing by means of quantitative model, the results showed that the number of fans and the average amount of forward had a significant effect on the microblog marketing effectiveness. By studying the present microblog marketing situation of a large insurance company, R.Daniel [3] concluded that different organizational strategies produce different marketing effect. B.Francesco [4] designed a set of heuristics Yahoo Meme social graph to simulate the real world, through the real record of the amount of Meme transmission parameters show the feasibility and effectiveness of microblog platform. Domestic movie microblog marketing research mainly focused on the microblog marketing methods and strategies. Yuming Fan [5] thought movie microblog marketing can be divided into Topics Marketing, Platform Marketing, and Word of Mouth Marketing and so on. He called for combination with the specific circumstances. Yiyang Zhao[6] analyzed the movie marketing strategy under the new media era and has been clear about the marketing concept and advantage. Jiru Fang[7] explored that how can small budget films develop effective movie marketing strategies on the basis of the long tail.

Based on these theories, the paper is going to study movie microblog marketing and set up a UID (User Information Define) deployment model to achieve high exposure and high conversion by the choice of the crowd and appropriate materials delivery.

\section{Microblog and Film Marketing}

Microblog, this social media platform makes film audience pay more attention to the film. It has become an important role in the movie marketing. Survey shows that more than 60 percent of cinema audiences have microblog accounts. Microblogging gives the film audience a better understanding of the movie and brings the increase of comment [8]. Microblog acts as a key 
intermediary in movie marketing process by reconcile controlled environment and uncontrollable environment.

\subsection{Film marketing service system based on microblog}

In film marketing service system based on microblog, microblog provides the producers/investors with movie promotion service, analysis of promotion effect, viewing population analysis and the analysis of box office appeal; For the issuer/movie cinemas, It provides reference analysis service, viewing population situation and the high box office appeal staffs analysis; For the viewers, It provides movie guides, information query, tickets and film critics service. The promotion of producers affect microblog discussion, microblog Reading affect the cinemas and cinemas' arrange results affect producers gain. So the interests' chain has been formed. As shown in Figure.1.

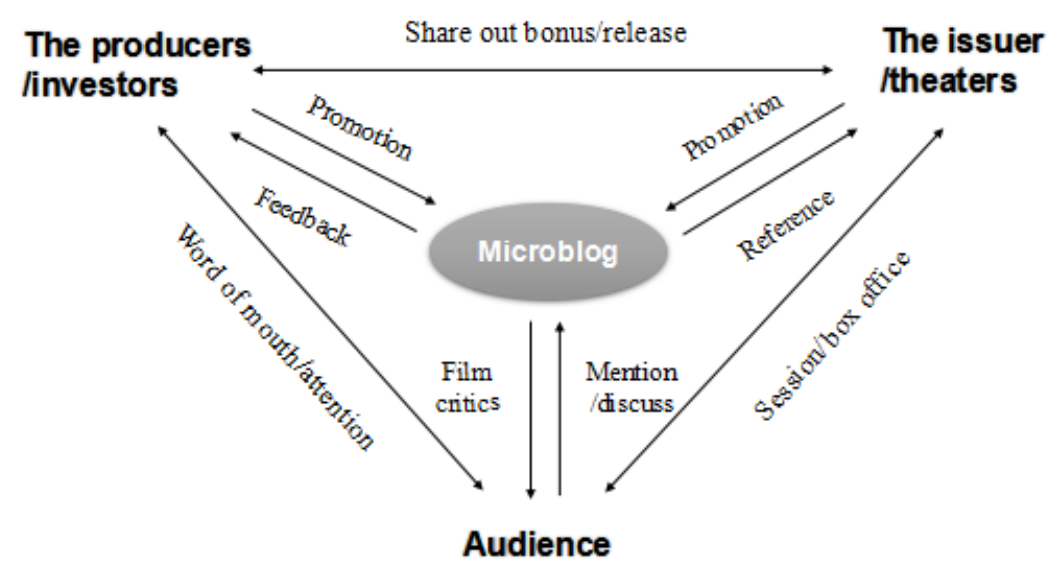

Fig. 1 Film marketing service system based on microblog

\subsection{Microblog precise delivery}

"Father" of department store John Wanamaker, once said: "I know that my AD half to waste, but unfortunately, I don't know which half is wasted." The traditional advertising face problems such as subscriptions decline. It gradually is shrinking for news content gatherer and advertising revenue decline year by year. The transparency of users' information in Internet advertising is low, so, it is hard to locate the user needs and can only carry on the extensive coverage. But, social media, which has interactive delivery, can be accurate to meet individual requirements. The advantage of social media is that through accurate contact and interaction it can quickly establish a user identification of the brand and further through users' purchase intention.

Microblog precision delivery is a kind of flexible delivery mode and it can realize the customization, high conversion and high exposure. To accurately target on the microblog, it needs to experience three stages: UID screening, UID dropping and effectiveness evaluation. Among them, the quality of UID screening, delivery time and content is particularly critical.

\section{The UID deployment model for microblog precision marketing}

The current movie advertising products mainly focus on information flow and these products are given priority to with users' management. The user has an independent data background for checking result data directly. This does not meet the demand for customized projects. Only real active users can be achieved on the content of the final arrival and exposure and only the right time and proper planning can ensure relevant content effective detonated in the accurate user groups, as the current product cannot fully be guaranteed.

Reference the process of microblog precise delivery, and comparison with the ordinary screening model of the current movie microblog marketing, this paper established a UID deployment model. In this model, different materials for different people can produce the same high ratio of investment products. As shown in Figure.2. 


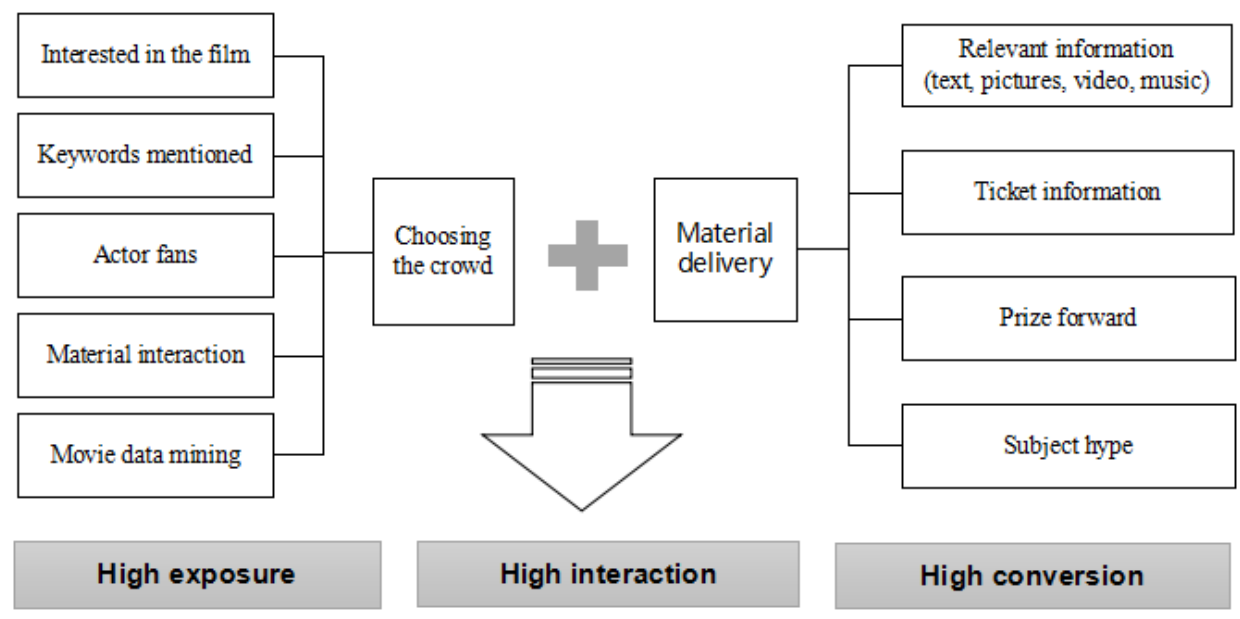

Fig. 2 The UID deployment model for microblog precision marketing

\subsection{Source of data extraction}

The user Library sources required as shown in Table.1.

Table. 1 Source of data extraction for UID deployment

\begin{tabular}{|c|c|c|}
\hline $\begin{array}{c}\text { Classificatio } \\
n\end{array}$ & Purpose & Data characteristics \\
\hline $\begin{array}{l}\text { Interested in } \\
\text { the film }\end{array}$ & $\begin{array}{l}\text { On the full } \\
\text { cover }\end{array}$ & $\begin{array}{l}\text { Huge, interest in precision, but there is no guarantee } \\
\text { that is interested in a particular movie }\end{array}$ \\
\hline $\begin{array}{l}\text { Keywords } \\
\text { mentioned }\end{array}$ & $\begin{array}{l}\text { Potential } \\
\text { interest }\end{array}$ & $\begin{array}{l}\text { User interest is relatively accurate, but the key word } \\
\text { set is critical, not sure orders of magnitude }\end{array}$ \\
\hline Actor fans & $\begin{array}{l}\text { High star } \\
\text { effect }\end{array}$ & $\begin{array}{l}\text { Affected by the amount of star fans, has to be active } \\
\text { and yuan distance filter can obtain high quality on } \\
\text { the object }\end{array}$ \\
\hline $\begin{array}{l}\text { Material } \\
\text { interaction }\end{array}$ & Direct interest & $\begin{array}{l}\text { Quantity is less and may overlap with fans to a } \\
\text { certain extent, the most direct interested in a } \\
\text { particular movie }\end{array}$ \\
\hline $\begin{array}{l}\text { Movie data } \\
\text { mining }\end{array}$ & $\begin{array}{l}\text { History of the } \\
\text { audience, a } \\
\text { specific type }\end{array}$ & $\begin{array}{l}\text { Can realize the movie, actor, director corresponding } \\
\text { to specific users }\end{array}$ \\
\hline
\end{tabular}

\subsection{The UID extraction rules and procedures}

For UID deployment requirements, formulate the UID extraction rules and processes as shown in figure.3. 


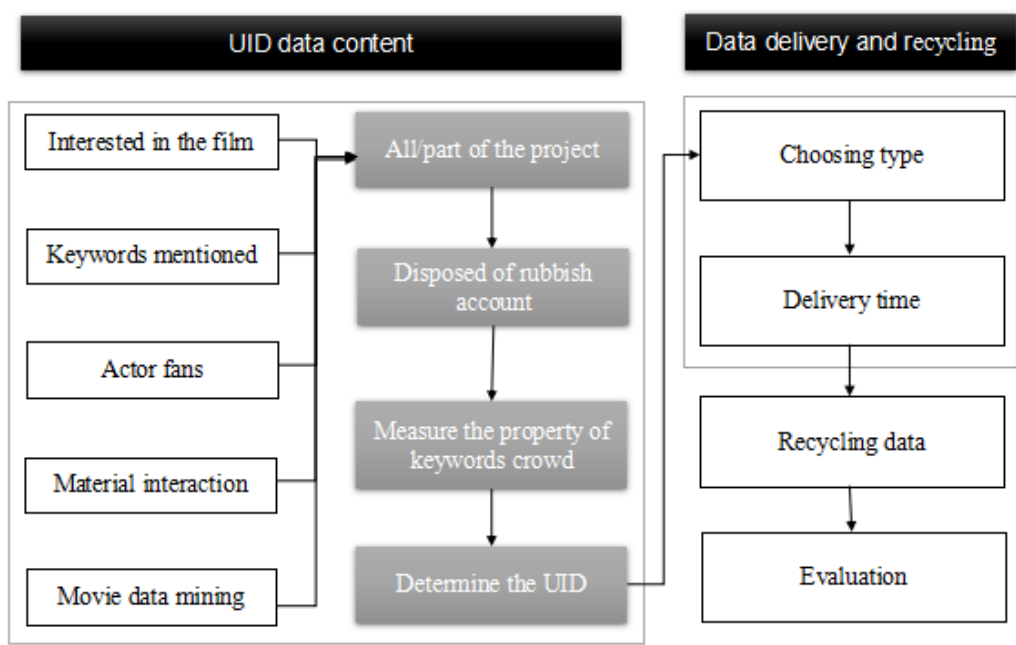

Fig. 3 The UID extraction rules and procedures

The recycling data index settings for UID deployment consists of basic data and derived indicators data. Basic indicators include exposure, the number of reading, the count, the comments, the thumb up forward, the number of collection, the short chain increase number of clicks and fans. Derived indicators include clickthrough rate, delivery efficiency and single cost.

\section{The UID deployment examples of "Breakup Buddies"}

The author had an internship in Sina Weibo (China's largest microblogging platform). At that time the above UID deployment model was used in the promotion for movie commercial project of "Breakup Buddies", and considerable data results have been achieved.

First of all, We determined film information relevant keywords (including film category, content, keywords, creators, fan interest, etc.) according to the film content of "Breakup Buddies", and then the background calculated and extracted the corresponding number mentioned to complete the UID preliminary selection. Next disposed of rubbish account is required. Then measured the property of keywords crowd and analyzed of human gender, age, province of login, user types, habits and interest preference, Finally the delivery UID extracted by the filter of active user time and user attribute information.

The process of extracting object keywords of "Breakup Buddies" is shown in figure.4.

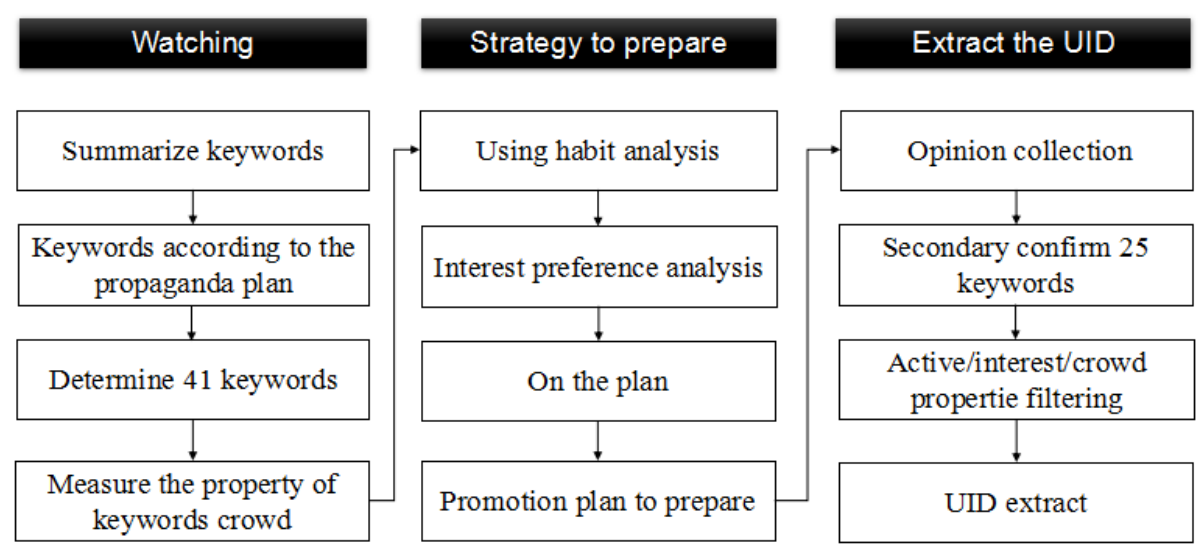

Fig. 4 The process of extracting object keywords of "Breakup Buddies"

The keywords combined with the film publicity plan are summarized in Table.2. 
Table. 2 examples of summarized keywords

\begin{tabular}{cccc}
\hline keywords & Mention & $\begin{array}{c}\text { Original } \\
\text { mention }\end{array}$ & Transpond mention \\
\hline Total & 32421188 & 5522693 & 26996104 \\
\hline funny & 6164358 & 976109 & 5207864 \\
Amuse & 6057392 & 478289 & 5607348 \\
Gay friends & 5538663 & 241749 & 5311335 \\
Prick silk & 4003542 & 1829391 & 2184622 \\
brokenhearted & 1855379 & 473430 & 1386108 \\
\hline
\end{tabular}

After determining the final delivery of the UID, via video information, ticketing information and prize forwarding we can verify that the Weibo Selection of accurate screening UID bring more than 60 times reading at ordinary times, 10 times than usual number of interactive, 9 times than usual exposure times for a single post. This model realizes the accurate touch up to the crowd and effective promotion and at the same time also proves the feasibility.

\section{Conclusion}

This paper studies the relationship between microblog and film marketing, noted the important role that the microblog played in the movie marketing service system. Among them, microblog precise delivery provides indispensable platform support for movies. However, the current movie advertising products mainly focus on information flow. It can't be ensured that related content can effectively transfer in the accurate user groups. Comparison with the ordinary screening model of the current movie microblog marketing, this paper established a UID deployment model, by people choose and appropriate materials delivery to achieve high exposure and high conversion.

The current study of microblog precision marketing for film industry is still in its infancy and there are many places need to be improved, such as improve mining dimension and precision and optimize effectiveness data collection process in order to get through "research, application, and then optimization".

\section{References}

[1] China Internet Network Information Center (CNNIC), 36 times China Internet network development state statistic report, 2015-7-23.

[2] Yaroslav Ryabov, An Empirical Research on Influencing Factors of Microblogging Marketing Effectiveness, Science and Technologs A.2013 (8): 06-04.

[3] R.Daniel, R.Alexander and H.Jakob, Infrastructures-in-practice: Cultivating enterprise microblogging, Proceedings of the Annual Hawaii International Conference on System Sciences, (2013), pp. 670-679.

[4] B.Francesco, C. Carlos and L. Dino, Meme ranking to maximize posts virality in microblogging platforms, Journal of Intelligent Information Systems, (2011), pp. 1-29.

[5] Yuming Fan, Research on Movie microblog marketing, Journal of southeast spread, 2012 (6).

[6] Yiyang Zhao, Microblog marketing strategy of small and medium-budget films under the new media age, Journal of news study Tribune, 2014, (7) : 05-07.

[7] Jiru Fang, Microblog marketing strategy of small and medium-budget films based on the theory of the long tail, Journal of broadcasting and television information, 2015.3.

[8] Yue Gao, Study of cinema audiences use of microblog and effects, 2012. 